\section{Sonazoid-enhanced ultrasonography: comparison with CT/MRI Liver Imaging Reporting and Data System in patients with suspected hepatocellular carcinoma}

\author{
Jeong Ah Hwang ${ }^{1}$, Woo Kyoung Jeong ${ }^{1}$, Ji Hye Min ${ }^{1}$, Yeun-Yoon $\mathrm{Kim}^{2}$, Nam Hun Heo³, \\ Hyo Keun Lim $^{1}$
}

${ }^{1}$ Department of Radiology and Center for Imaging Science, Samsung Medical Center, Sungkyunkwan University School of Medicine, Seoul; ${ }^{2}$ Department of Radiology and Research Institute of Radiological Science, Severance Hospital, Yonsei University College of Medicine, Seoul; ${ }^{3}$ Clinical Trial Center, Soonchunhyang University Cheonan Hospital, Soonchunhyang University College of Medicine, Cheonan, Korea

Purpose: The aim of this study was to evaluate the association of contrast-enhanced ultrasound (CEUS) features using Sonazoid for liver nodules with Liver Imaging Reporting and Data System (LI-RADS) categories and to identify the usefulness of Kupffer-phase images.

Methods: This retrospective study was conducted in 203 patients at high risk of hepatocellular carcinoma (HCC) who underwent CEUS with Sonazoid from 2013 to 2016. Nodule enhancement in the arterial, portal venous, late, and Kupffer phases; CEUS LI-RADS major features; and Kupffer-phase defects were evaluated. According to the computed tomography/magnetic resonance imaging (CT/MRI) LI-RADS V2018, all nodules were assigned an LR category ( $n=4 / 33 / 99 / 67$ for LR-M/3/4/5) and comparisons across LR categories were made. We defined modified CEUS LI-RADS as using Kupffer-phase defects as an alternative to late and mild washout in CEUS LI-RADS and compared the diagnostic performance for HCC.

Results: On CEUS of 203 nodules, $89.6 \%$ of CT/MRI LR-5 and $85.9 \%$ of LR-4 nodules showed hyperenhancement in the arterial phase, while $57.6 \%$ of LR-3 nodules showed hyperenhancement. Among the CT/MRI LR-5 nodules that showed arterial phase hyperenhancement or isoenhancement, $59.7 \%$ showed hypoenhancing changes from the portal venous phase, $23.9 \%$ from the late phase, and $13.4 \%$ additionally in the Kupffer phase. The modified CEUS LI-RADS showed higher sensitivity than CEUS LI-RADS (83.2\% vs. $74.2 \%$, $\mathrm{P}=0.008)$ without compromising specificity $(63.6 \%$ vs. $69.7 \%, \mathrm{P}=0.500)$.

Conclusion: The Kupffer phase best shows hypoenhancing changes in LR-5 lesions and is expected to improve the sensitivity for HCC in high-risk patients.

Keywords: Ultrasonography; Contrast media; Kupffer cells; Liver neoplasms; Carcinoma, hepatocellular

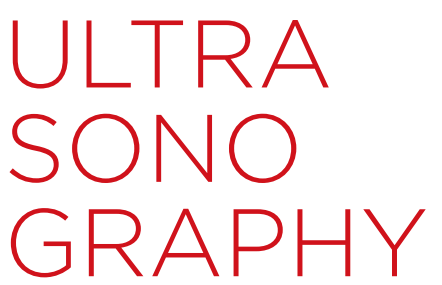

ORIGINAL ARTICLE

https://doi.org/10.14366/usg.20120 pISSN: 2288-5919 • elSSN: 2288-5943 Ultrasonography 2021;40:486-498

Received: August 5, 2020 Revised: January 14, 2021 Accepted: January 15, 2021

Correspondence to: Woo Kyoung Jeong, MD, Department of Radiology and Center for Imaging Science, Samsung Medical Center, Sungkyunkwan University School of Medicine, 81 Irwon-ro, Gangnam-gu, Seoul 06351, Korea

Tel. +82-2-3410-1923

Fax. +82-2-3410-0084

E-mail: jeongwk@gmail.com

This is an Open Access article distributed under the terms of the Creative Commons Attribution NonCommercial License (http://creativecommons.org/ licenses/by-nc/4.0/) which permits unrestricted noncommercial use, distribution, and reproduction in any medium, provided the original work is properly cited.

Copyright @ 2021 Korean Society of Ultrasound in Medicine (KSUM)

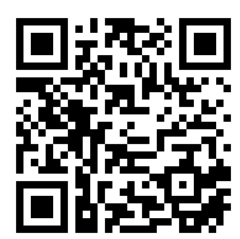

How to cite this article:

Hwang JA, Jeong WK, Min JH, Kim YY, Heo NH, Lim HK. Sonazoid-enhanced ultrasonography: comparison with CT/MRI Liver Imaging Reporting and Data System in patients with suspected hepatocellular carcinoma. Ultrasonography. 2021 0ct;40(4):486-498. 


\section{Introduction}

Sonazoid (GE Healthcare, Oslo, Norway), is a second-generation ultrasound contrast agent approved in Japan, Denmark, Norway, and South Korea for use in contrast-enhanced ultrasonography (CEUS) of focal liver lesions [1]. Sonazoid consists of microbubbles of perfluorobutane gas with phospholipid monolayer shells. CEUS using Sonazoid enables imaging of the vascular phase and the post-vascular Kupffer phase (KP) [2]. Hepatic enhancement in the $\mathrm{KP}$ may be due to the phagocytosis of microbubbles by Kupffer cells (liver-specific macrophages), adherence of microbubbles to the liver sinusoids and tumor vascular spaces, or recirculation of microbubbles [3-5]. The American College of Radiology released the 2017 version of the CEUS Liver Imaging Reporting and Data System (LI-RADS) for focal liver lesion assessment using only pure bloodpool agents [1]. The current version of the LI-RADS does not address the use of combined blood-pool and Kupffer cell agents such as Sonazoid, although this issue is scheduled to be included in the next revision.

The Kupffer cell count reflects the progression of hepatocarcinogenesis from dysplastic nodule (DN) to overt hepatocellular carcinoma (HCC) $[6,7]$. It plays an important role in both the diagnosis of HCC and the differentiation of well-differentiated early HCC and DNs [8]. Active research has been recently conducted using Sonazoidenhanced ultrasonography (US) to characterize focal liver tumors [9-11], revealing correlations between tumor differentiation and CEUS findings $[6,12,13]$. Sonazoid-enhanced US has the potential to assist in the evaluation of the post-treatment response $[14,15]$ and to serve as a guide for treatment approach [16] or act as a surveillance test for HCC [17]. Nevertheless, the imaging features at each phase of Sonazoid-enhanced US for liver nodules suspected of HCC have not been well studied. Previous research has evaluated CEUS features using Sonazoid for HCC compared with computed tomography (CT) features; however, only the early vascular phase and KP were assessed [18]. Moreover, few studies have been published on CEUS features using Sonazoid for each CT/magnetic resonance imaging (MRI) LI-RADS category.

This study aimed to evaluate the associations of CEUS features using Sonazoid for liver nodules, including the major features of CEUS LI-RADS, with CT/MRI LI-RADS categories and to determine the usefulness of KP images in the diagnosis of HCC.

\section{Materials and Methods}

\section{Compliance with Ethical Standards}

This retrospective study received Institutional Review Board approval at Samsung Medical Center in Korea (SMC 2019-07-147) and the requirement for informed consent was waived.

\section{Study Population}

Between January 2013 and December 2016, 334 patients underwent CEUS using Sonazoid at our institution as a second round of imaging after an inconclusive diagnosis, for pre-treatment assessment before locoregional therapy, or for characterization of a focal liver lesion found by conventional US. Among them, 258 patients were enrolled according to the following inclusion criteria: (1) patients at high risk of HCC, and (2) patients who had available liver CT/MRI information within 3 months of the CEUS exam. Fifty-
334 Patients underwent CEUS using Sonazoid between January 2013 and December 2016

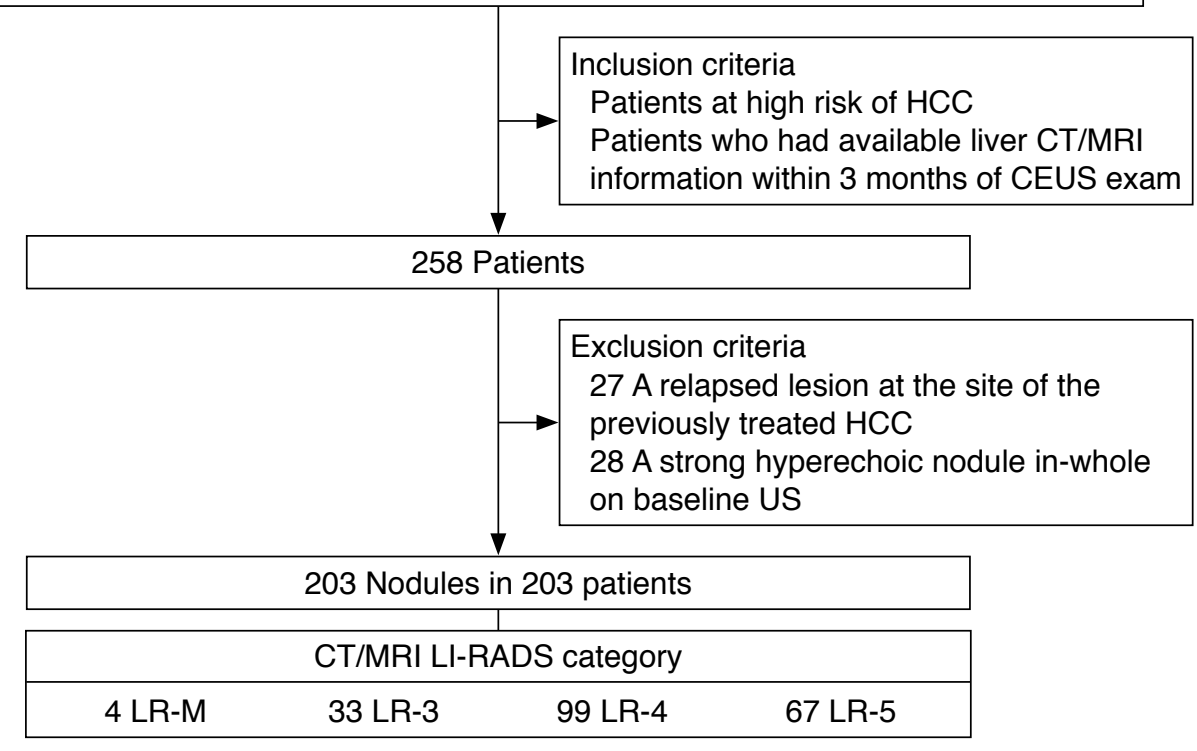

Fig. 1. Flow diagram of patient selection. CEUS, contras t-enhanced ultrasonography; HCC, hepatocellular carcinoma; CT, computed tomography; MRI, magnetic resonance imaging; US, ultrasonography; LI-RADS (LR), Liver Imaging Reporting and Data System. 


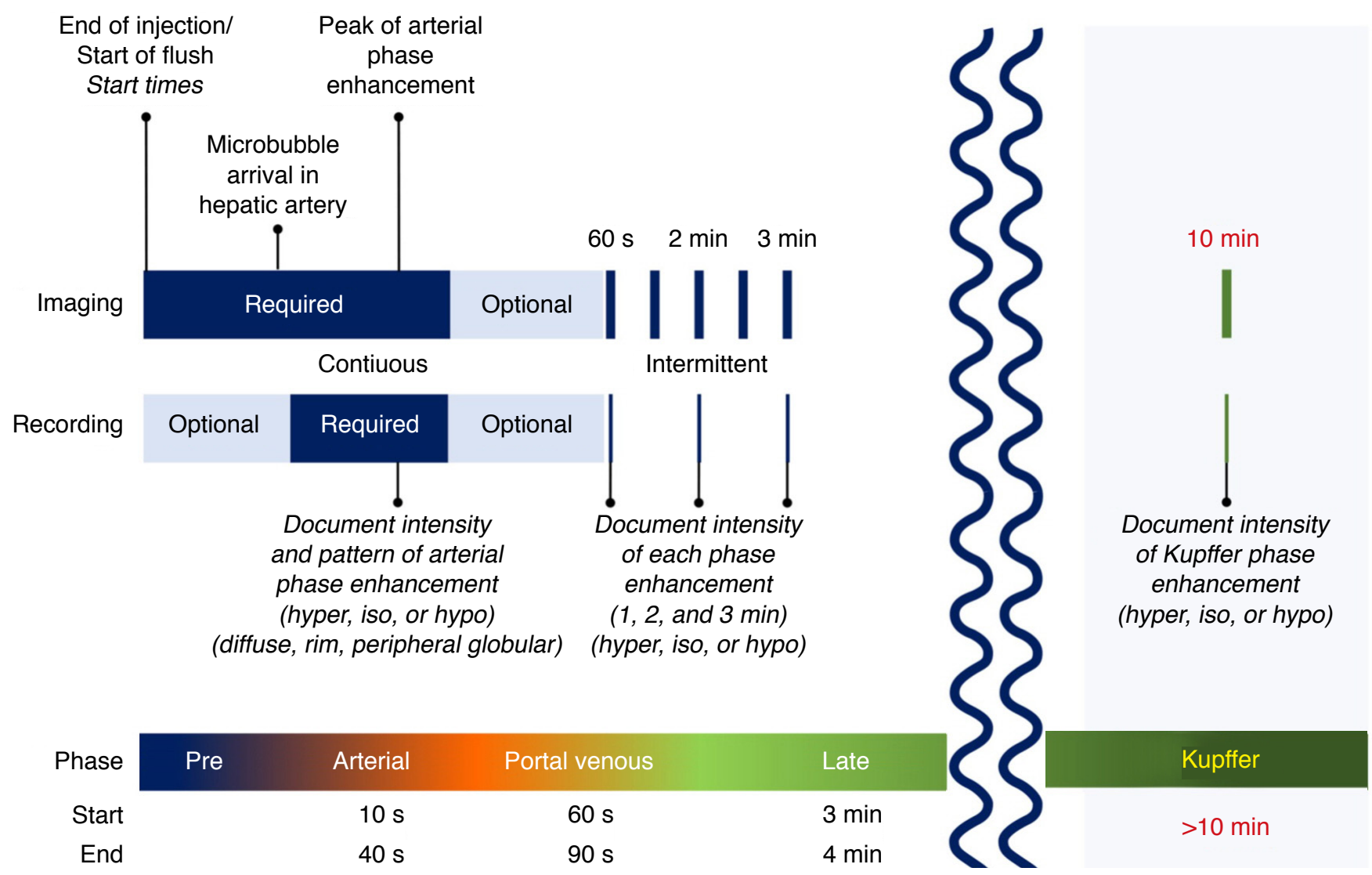

Fig. 2. The imaging acquisition protocol of Sonazoid-enhanced ultrasonography. The timer was started after the end of contrast injection/ start of the saline flush. The vascular phase images were acquired during arterial, portal venous, and late phases, at 10-40 seconds, at 6090 seconds, and at 3-4 minutes after contrast injection, respectively. Continuous imaging, as a cine loop, was acquired during the peak of arterial phase enhancement. Optionally, the cine loop could be continued before and after the peak of arterial phase enhancement. Intermittent imaging, comprising a series of brief image acquisitions (each lasting a few seconds and spaced apart without imaging in between), was acquired every 60 seconds. Approximately 10 minutes after contrast injection, Kupffer-phase images were obtained for several seconds. The intensity and pattern of arterial phase enhancement, intensity of portal venous, late and Kupffer-phase enhancement, and time of washout onset (if any) were documented.

five patients were excluded due to (1) a relapsed lesion at the site of the previously treated HCC (local tumor progression), or (2) a strongly hyperechoic nodule in-whole on baseline US. Despite a contrast-specific subtraction technique known as pulse inversion harmonic imaging, there could be limitations in the assessment of contrast enhancement and washout in Sonazoid-enhanced US at a low mechanical index for hyperechoic nodules on background grayscale US [19-21]. Partly hyperechoic nodules, the nonhyperechoic portions of which could be analyzed, were included. Ultimately, 203 nodules in 203 patients were included in this study (Fig. 1).

\section{CEUS Examinations}

Sonazoid-enhanced US examinations were conducted using LOGIQ E9 (GE Healthcare, Milwaukee, WI, USA) with a 3-5 MHz curved array transducer immediately after grayscale US; $16 \mu \mathrm{g}$ of perfluorobutane in one vial of Sonazoid was dissolved in $2 \mathrm{~mL}$ of water. This solution was intravenously administrated into the antecubital vein as a bolus at a dose of $0.015 \mathrm{~mL} / \mathrm{kg}$ body weight, followed by a 10-mL saline flush. If the lesion was observed with poor conspicuity on grayscale or if the examiner requested it, the imaging fusion technique of CT/MRI with US was used.

The imaging protocol is summarized in Fig. 2 [21]. Grayscale (or CT/MRI fusion) and contrast-mode images were viewed on dualscreen displays and the scanner timer was used to record the time from contrast injection for all images. The timer was started after the end of contrast injection/start of saline flush. The vascular phase images were acquired during the arterial phase (AP; 10-40 seconds after contrast injection), the portal venous phase (PVP; 60 seconds after contrast injection), and the late phase (LP; 3 minutes after contrast injection). Continuous imaging, as a cine loop, was acquired during the peak of AP enhancement with the option to continue 
Table 1. Baseline characteristics

\begin{tabular}{|c|c|}
\hline Variable & Value \\
\hline Per-patient & 203 \\
\hline Male sex & $159(78.3)$ \\
\hline Age (year) & $61.3 \pm 9.5(32-83)$ \\
\hline \multicolumn{2}{|l|}{ Etiology of chronic liver disease } \\
\hline Hepatitis B & $171(84.2)$ \\
\hline Hepatitis C & $17(8.4)$ \\
\hline Hepatitis B and C & $5(2.5)$ \\
\hline Alcohol & $7(3.4)$ \\
\hline Unknown & $3(1.5)$ \\
\hline Liver cirrhosis & $155(76.4)$ \\
\hline HCC diagnosis history & $195(96.1)$ \\
\hline Per-lesion & 203 \\
\hline \multicolumn{2}{|l|}{ Nodule echogenicity on grayscale US } \\
\hline Hypoechoic & $141(69.5)$ \\
\hline Isoechoic & $62(30.5)$ \\
\hline Size on grayscale US (cm) & $1.5 \pm 0.7(0.7-5.0)$ \\
\hline \multicolumn{2}{|l|}{ Diagnosis method } \\
\hline Biopsy & $7(3.5)$ \\
\hline Surgery & $24(11.8)$ \\
\hline Imaging diagnosis & $172(84.7)$ \\
\hline \multicolumn{2}{|l|}{ CT/MRI LI-RADS category } \\
\hline LR-M & $4(2.0)$ \\
\hline LR-3 & $33(16.3)$ \\
\hline LR-4 & $99(48.8)$ \\
\hline LR-5 & $67(33.0)$ \\
\hline Histologically proven lesions & $31(15.3)$ \\
\hline $\mathrm{HCC}$ & $23(74.2)$ \\
\hline $\begin{array}{l}\text { Hepatocellular nodule with fatty change (about } \\
30 \%)\end{array}$ & $1(3.2)$ \\
\hline Regenerative nodule & $1(3.2)$ \\
\hline Focal nodular hyperplasia & $3(9.7)$ \\
\hline Reactive lymphoid hyperplasia & $1(3.2)$ \\
\hline Sclerosing hemangioma & $1(3.2)$ \\
\hline Old and fresh hemorrhage & $1(3.2)$ \\
\hline
\end{tabular}

Values are presented as number (\%) or mean \pm SD (range).

HCC, hepatocellular carcinoma; US, ultrasonography; CT, computed tomography; $\mathrm{MRI}$, magnetic resonance imaging; LI-RADS (LR), Liver Imaging Reporting and Data System; SD, standard deviation.

the cine loop before and after the peak, especially if tumor washout within $<60$ seconds was found. Intermittent imaging, comprising a series of brief image acquisitions (each lasting a few seconds and spaced apart without imaging in between), was acquired every 60 seconds. Approximately 10 minutes after contrast injection, KP images were obtained for several seconds. The scanning parameters are detailed in Supplementary Table 1.

\section{CEUS Imaging Assessment}

Two abdominal radiologists (J.A.H. and W.K.J. with 9 and 20 years of experience in abdominal imaging, respectively) independently reviewed the recorded CEUS video clips and static images. Discordances were resolved by consensus through discussions with a third radiologist (H.K.L. with 35 years of experience in abdominal imaging). The reviewers were blinded to the CT/MRI LR categories and histological diagnoses of each case.

The following imaging features were evaluated: (1) nodule echogenicity and size $(\mathrm{cm})$ on grayscale; (2) nodule enhancement in the AP, PVP, LP, and KP (hyperenhancement, isoenhancement, or hypoenhancement compared to the liver in each phase); (3) pattern of wash-in (i.e., in-whole or in-part, rim, stellate vessels, or peripheral globular) in the AP if applicable; (4) if and when (i.e., within 60 seconds after contrast injection, during the PVP, $L P$, or KP) washout occurred (a temporal reduction in enhancement of a nodule in-whole or in-part relative to the liver after peak AP enhancement [1]); (5) major features according to CEUS LI-RADS v2017 including arterial phase hyperenhancement (APHE; a lesion becoming hyperenhancing in-whole or in-part compared to the liver in the AP that is neither rim-like nor peripheral globular) and late and mild washout (a lesion that becomes hypoenhancing compared to the liver in the PVP and LP, occurring $>60$ seconds from contrast injection, and without marked hypoenhancement or a punchedout appearance within 2 minutes after contrast injection) [22]; (6) $\mathrm{KP}$ defect (a lesion that is hypoenhancing compared to the liver in the KP [Fig. 3]); (7) ancillary features according to CEUS LI-RADS v2017 including the nodule-in-nodule sign (smaller inner nodules that differ from the larger outer nodules) and mosaic architecture (presence of randomly distributed internal nodules or compartments) [22]; (8) LR-M criteria according to CEUS LI-RADS v2017 including rim APHE (most pronounced AP enhancement in the periphery of the observation), early washout (within 60 seconds from contrast injection), or marked washout (observation appears black or punched out within 2 minutes after contrast injection) [22]. If two or more lesions were present in a single patient, a histologically confirmed lesion or the largest lesion was selected as the target lesion.

According to the CEUS LI-RADS V2017, all of the nodules were assigned to an LR category based on APHE, the nodule size, and late and mild washout. We defined a modified CEUS LI-RADS using the criterion of a KP defect as an alternative to late and mild washout and assigned LR categories according to the modified CEUS LIRADS. When a nodule demonstrated nodule-in-nodule or mosaic architecture, the LR category was upgraded by one category up to 


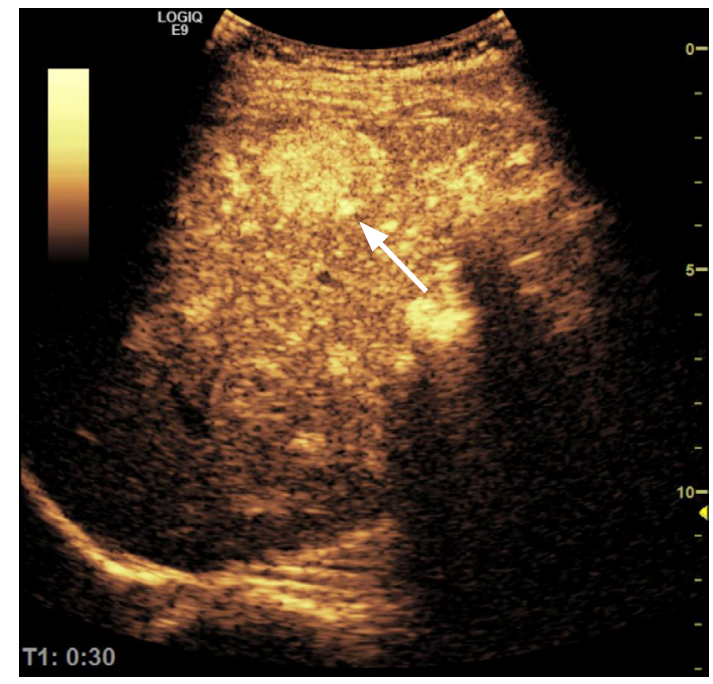

A

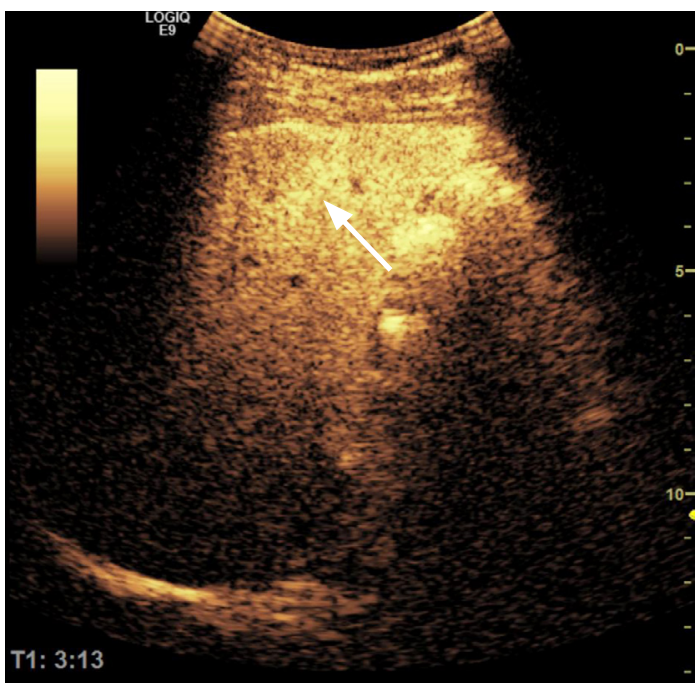

C

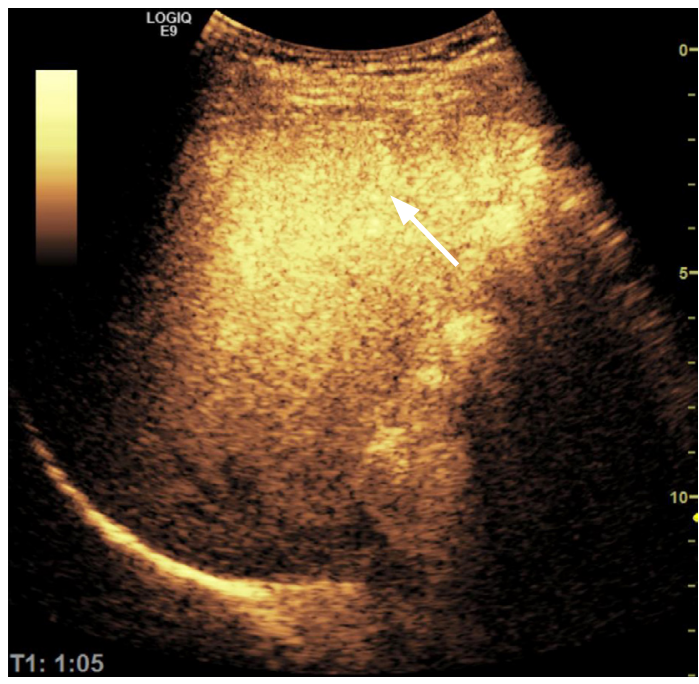

B

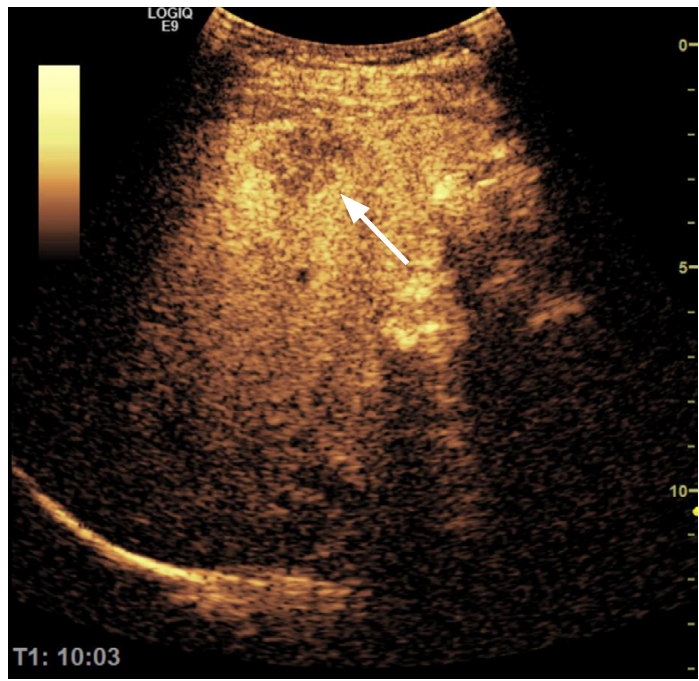

D

Fig. 3. Sonazoid-enhanced US with imaging fusion for a CT/MRI LI-RADS LR-5 nodule in a 68-year-old man with chronic viral hepatitis B. A. Arterial phase image shows a 3.2-cm hyperenhancing liver nodule (arrow) in hepatic segment $\mathrm{V}$. B. At 1 minute after injection, washout has not occurred yet (arrow). C. After 3 minutes, a subtle hypoenhancing lesion (arrow) is seen, but is not obvious. D. On the Kupfferphase image, hypoenhancement in the nodule (arrow) is more conspicuous. US, ultrasonography; CT, computed tomography; MRI, magnetic resonance imaging; LI-RADS (LR), Liver Imaging Reporting and Data System.

LR-4 in both CEUS LI-RADS and the modified CEUS LI-RADS [23].

\section{CT/MRI Imaging Assessment}

A radiologist (J.H.M. with 11 years of experience in abdominal imaging) who was blinded to the CEUS findings analyzed the CT or MRI images of all 203 nodules, and assigned them to LI-RADS category according to CT/MRI LI-RADS v2018 [24]. The ancillary features and tie-breaking rules were applied. For patients with gadoxetic acid-enhanced MRI, signal intensity of the nodule in the hepatobiliary phase (HBP) was also assessed. To use as a reference standard for evaluating the diagnostic performance of CEUS for HCC, subjects were allocated into HCC and non-HCC groups based on histology, whenever available, or a CT/MRI imaging diagnosis of HCC according to the Korean Liver Cancer Association-National Cancer Center (KLCA-NCC) Korea Practice Guidelines [25]. Since the American Association for the Study of Liver Diseases and European Association for the Study of the Liver, as well as the KLCA-NCC, do not allow the imaging diagnosis of sub-centimeter-sized HCCs, an 
Table 2. Sonazoid-enhanced US features of 203 hepatic nodules by CT/MRI LI-RADS category

\begin{tabular}{|c|c|c|c|c|c|}
\hline & \multirow{2}{*}{ Total } & \multicolumn{4}{|c|}{ CT/MRI LI-RADS category } \\
\hline & & LR-M $(n=4)$ & LR-3 $(n=33)$ & LR-4 ( $n=99)$ & $\operatorname{LR}-5(n=67)$ \\
\hline \multicolumn{6}{|l|}{ AP } \\
\hline Hyperenhancement & 168 & $4(100)$ & 19 (57.6) & $85(85.9)$ & 60 (89.6) \\
\hline Isoenhancement & 28 & 0 & 11(33.3) & $11(11.1)$ & $6(9.0)$ \\
\hline Hypoenhancement & 7 & 0 & $3(9.1)$ & $3(3.0)$ & $1(1.5)$ \\
\hline \multicolumn{6}{|l|}{ PVP } \\
\hline Hyperenhancement & 6 & $1(25.0)$ & $3(9.1)$ & $1(1.0)$ & $1(1.5)$ \\
\hline Isoenhancement & 85 & 0 & $19(57.6)$ & $41(41.4)$ & $25(37.3)$ \\
\hline Hypoenhancement & 112 & $3(75.0)$ & $11(33.3)$ & $57(57.6)$ & $41(61.2)$ \\
\hline \multicolumn{6}{|l|}{ LP } \\
\hline Hyperenhancement & 1 & 0 & $1(3.0)$ & 0 & 0 \\
\hline Isoenhancement & 36 & 0 & $13(39.4)$ & $13(13.1)$ & $10(14.9)$ \\
\hline Hypoenhancement & 166 & $4(100)$ & $19(57.6)$ & $86(86.9)$ & $57(85.1)$ \\
\hline \multicolumn{6}{|l|}{ KP } \\
\hline Hyperenhancement & 0 & 0 & 0 & 0 & 0 \\
\hline Isoenhancement & 16 & 0 & $8(24.2)$ & $7(7.1)$ & $1(1.5)$ \\
\hline Hypoenhancement & 187 & $4(100)$ & $25(75.8)$ & $92(92.9)$ & $66(98.5)$ \\
\hline \multicolumn{6}{|l|}{ Pattern of wash-in } \\
\hline In-whole or in-part & 163 & $2(50.0)$ & $17(51.5)$ & $85(85.9)$ & $59(88.1)$ \\
\hline $\operatorname{Rim}$ & 2 & $2(50.0)$ & 0 & 0 & 0 \\
\hline Stellate vessels & 3 & 0 & $2(6.1)$ & 0 & $1(1.5)$ \\
\hline Not applicable & 35 & 0 & $14(42.4)$ & $14(14.1)$ & $7(10.4)$ \\
\hline \multicolumn{6}{|l|}{ Washout first appeared } \\
\hline Hyper-/isoenhancement in AP & 196 & & & & \\
\hline Within $60 \mathrm{~s}$ & 1 & $1(25.0)$ & 0 & 0 & 0 \\
\hline From PVP & 104 & $2(50.0)$ & $8(24.2)$ & $54(54.5)$ & $40(59.7)$ \\
\hline From LP & 54 & $1(25.0)$ & $8(24.2)$ & $29(29.3)$ & $16(23.9)$ \\
\hline From KP & 21 & 0 & $6(18.2)$ & $6(6.1)$ & $9(13.4)$ \\
\hline No washout & 16 & 0 & $8(24.2)$ & $7(7.1)$ & $1(1.5)$ \\
\hline Hypoenhancement in all phase & 7 & 0 & $3(9.1)$ & $3(3.0)$ & $1(1.5)$ \\
\hline \multicolumn{6}{|l|}{ CEUS LR-M criteria } \\
\hline Rim APHE & 2 & $2(50.0)$ & 0 & 0 & 0 \\
\hline Early washout & 1 & $1(25.0)$ & 0 & 0 & 0 \\
\hline Marked washout & 1 & $1(25.0)$ & 0 & 0 & 0 \\
\hline
\end{tabular}

Values are presented as number (\%).

US, ultrasonography; CT, computed tomography; MRI, magnetic resonance imaging; LI-RADS (LR), Liver Imaging Reporting and Data System; AP, arterial phase; PVP, portal venous phase; LP, late phase; KP, Kupffer phase; CEUS, contrast-enhanced ultrasonography; APHE, arterial phase hyperenhancement.

imaging diagnosis of HCC was only conducted for lesions of $1 \mathrm{~cm}$ or more.

\section{Statistical Analysis}

Continuous variables were reported as mean \pm standard deviation and range. Descriptive data were reported as numbers and percentages. Comparisons across groups were made using the chisquare test or Fisher exact test. For hepatic nodules $\geq 1 \mathrm{~cm}$, the diagnostic performance of CEUS and modified CEUS LI-RADS was evaluated using the HCC group as the reference standard and compared using the McNemar test or Delong's method. The analysis was performed using SPSS version 25 (IBM Corp., Armonk, NY, USA). 
A P-value $<0.05$ was considered to indicate statistical significance.

\section{Results}

\section{Baseline Characteristics of the Study Population}

A total of 203 patients (mean age, $61.3 \pm 9.5$ years; age range, 32 to 83 years: 159 men [mean age, $60.6 \pm 9.0$ years; age range, 34 to 79 years]: 44 women [mean age, $63.2 \pm 10.9$ years; age range, 32 to 83 years]) were included. The baseline characteristics of the

Table 3. Comparison of CEUS LI-RADS major and ancillary features and Kupffer-phase defects of 199 hepatic nodules in CT/MRI LR-3, LR-4, and LR-5 on Sonazoid-enhanced US

\begin{tabular}{|c|c|c|c|c|c|}
\hline & \multirow[b]{2}{*}{ Total } & \multicolumn{4}{|c|}{ CT/MRI LI-RADS category } \\
\hline & & $\begin{array}{c}\text { LR-3 } \\
(n=33)\end{array}$ & $\begin{array}{c}\text { LR-4 } \\
(n=99)\end{array}$ & $\begin{array}{c}\text { LR-5 } \\
(n=67)\end{array}$ & P-value \\
\hline $\begin{array}{l}\text { Arterial phase } \\
\text { hyperenhancement }\end{array}$ & 164 & 19 (57.6) & $85(85.9)$ & 60 (89.6) & $<0.001$ \\
\hline Late and mild washout & 155 & $16(48.5)$ & $83(83.8)$ & $56(83.6)$ & $<0.001$ \\
\hline Kupffer-phase defect & 183 & $25(75.8)$ & $92(92.9)$ & $66(98.5)$ & $<0.001$ \\
\hline $\begin{array}{l}\text { Nodule-in-nodule or } \\
\text { mosaic }\end{array}$ & 28 & $3(9.1)$ & $10(10.1)$ & $15(22.4)$ & 0.056 \\
\hline
\end{tabular}

Values are presented as number (\%).

CEUS, contrast-enhanced ultrasonography; LI-RADS (LR), Liver Imaging Reporting and Data System; CT, computed tomography; MRI, magnetic resonance imaging; US, ultrasonography.

Table 4. Nodule classification according to CEUS and modified CEUS LI-RADS for 122 hepatic nodules $\geq 1 \mathrm{~cm}$

\begin{tabular}{ccc} 
& \multicolumn{2}{c}{ LR classification } \\
\cline { 2 - 3 } & CEUS LI-RADS & Modified CEUS LI-RADS \\
\hline LR-M & $1(0.8)$ & $1(0.8)$ \\
LR-3 & $20(16.4)$ & $20(16.4)$ \\
LR-4 & $25(20.5)$ & $15(12.3)$ \\
LR-5 & $76(62.3)$ & $86(70.5)$ \\
\hline
\end{tabular}

Values are presented as number (\%).

CEUS, contrast-enhanced ultrasonography; LI-RADS (LR), Liver Imaging Reporting and Data System. patients and nodules are summarized in Table 1. On grayscale US images, 141 nodules (69.5\%) were hypoechoic and 62 nodules (30.5\%) were isoechoic. The mean nodule size on grayscale US was $1.5 \pm 0.7 \mathrm{~cm}$ (range, 0.7 to $5.0 \mathrm{~cm}$ ). All nodules were assigned to LR categories according to CT/MRI LI-RADS V2018 as follows: four nodules $(2.0 \%)$ as LR-M, 33 nodules $(16.3 \%)$ as LR-3, 99 nodules $(48.8 \%)$ as LR-4, and 67 nodules (33.0\%) as LR-5. Thirty-one nodules had histological diagnoses and among these, 23 nodules (74.2\%) were HCCs.

Table 6. Comparison of nodule enhancement in MRI hepatobiliary phase and CEUS Kupffer phase images

\begin{tabular}{|c|c|c|c|c|}
\hline \multirow{2}{*}{ Kupffer phase } & \multirow{2}{*}{ No. } & \multicolumn{2}{|c|}{ Hepatobiliary phase } & \multirow{2}{*}{ P-value } \\
\hline & & Iso-/Hyperintensity & Hypointensity & \\
\hline $\begin{array}{l}\text { CT/MRI LR-3, LR-4, and } \\
\text { LR-5 }\end{array}$ & 193 & 9 & 184 & \\
\hline Isoenhancement & 15 & $3(33.3)$ & $12(6.5)$ & 0.024 \\
\hline Hypoenhancement & 178 & $6(66.7)$ & $172(93.5)$ & \\
\hline CT/MRI LR-3 & 32 & 6 & 26 & \\
\hline Isoenhancement & 8 & $3(50.0)^{\mathrm{a})}$ & $5(19.2)$ & \\
\hline Hypoenhancement & 24 & $3(50.0)^{b)}$ & $21(80.8)$ & \\
\hline CT/MRI LR-4 & 97 & 2 & 95 & \\
\hline Isoenhancement & 6 & 0 & $6(6.3)$ & \\
\hline Hypoenhancement & 91 & $2(100)^{c)}$ & $89(93.7)$ & \\
\hline CT/MRI LR-5 & 64 & 1 & 63 & \\
\hline Isoenhancement & 1 & 0 & $1(1.6)$ & \\
\hline Hypoenhancement & 63 & $1(100)^{d)}$ & $62(98.4)$ & \\
\hline
\end{tabular}

\section{Values are presented as number (\%).}

MRI, magnetic resonance imaging; CEUS, contrast-enhanced ultrasonography; $C T$, computed tomography; LR, Liver Imaging Reporting and Data System category.

${ }^{a)} A$ total of six patients were excluded, including three who underwent MRI with extracellular contrast agent and three who underwent CT without MRI. Three hyperintense nodules in the hepatobiliary phase. ${ }^{\text {b) }}$ Three isointense nodules in the hepatobiliary phase. ${ }^{c}$ One isointense and one hyperintense nodules in the hepatobiliary phase. ${ }^{d)}$ One isointense nodule in the hepatobiliary phase. The Fisher exact test was performed.

Table 5. Comparison of the diagnostic performance of CEUS and modified CEUS LI-RADS for 122 hepatic nodules $\geq 1 \mathrm{~cm}$

\begin{tabular}{lccccccc}
\hline & \multicolumn{5}{c}{ Diagnostic performance } \\
\cline { 2 - 7 } & Sensitivity (\%) & Specificity (\%) & Accuracy (\%) & AUC (\%) & LR+ & LR- & dOR \\
\hline Modified CEUS LI-RADS & $83.2(73.7-90.3)$ & $63.6(45.1-79.6)$ & $77.9(69.5-84.9)$ & $73.4(64.2-82.6)$ & $2.3(1.4-3.6)$ & $0.3(0.2-0.4)$ & $8.6(3.5-21.2)$ \\
CEUS LI-RADS & $74.2(63.8-82.9)$ & $69.7(51.3-84.4)$ & $73.0(64.2-80.6)$ & $71.9(62.8-81.1)$ & $2.4(1.4-4.2)$ & $0.4(0.2-0.6)$ & $6.6(2.7-15.9)$ \\
P-value & $0.008^{\text {a) }}$ & $0.500^{\text {a) }}$ & $0.109^{\text {a) }}$ & $0.574^{\text {b) }}$ & & & \\
\hline
\end{tabular}

Data in parentheses are $95 \%$ confidence intervals.

CEUS, contrast-enhanced ultrasonography; LI-RADS, Liver Imaging Reporting and Data System; AUC, area under the curve; LR+, positive likelihood ratio; LR-, negative likelihood ratio; dOR, diagnostic odds ratio.

${ }^{\text {a) }}$ McNemar test. belong's method. 


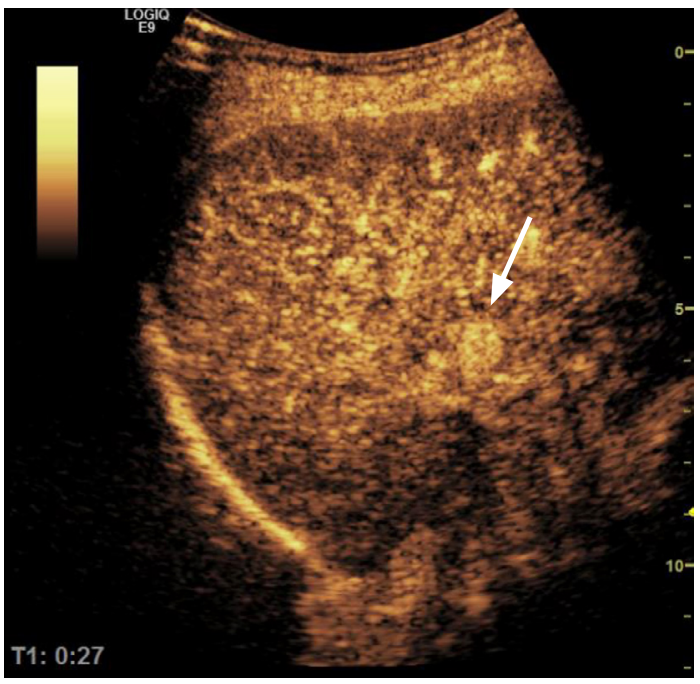

A

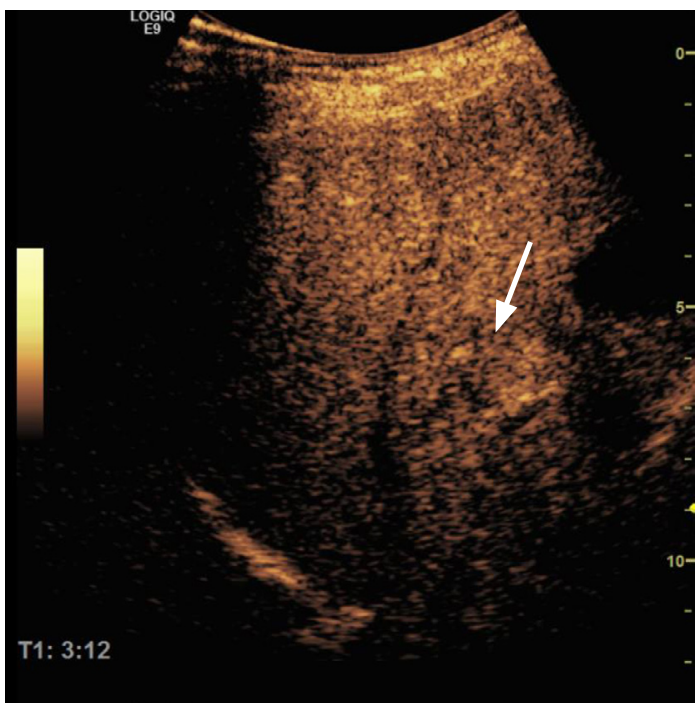

C



B

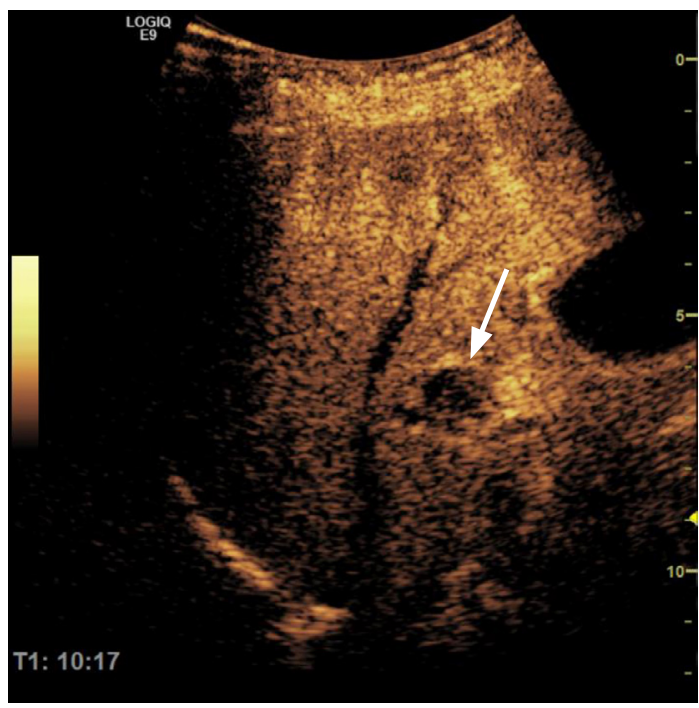

D

Fig. 4. Sonazoid-enhanced US with imaging fusion for a CT/MRI LI-RADS LR-5 nodule in a 68-year-old woman with chronic viral hepatitis $B$.

A. Arterial phase image shows a 1.2-cm hyperenhancing liver nodule (arrow) in hepatic segment VII. B, C. In portal venous and late phase images, no hypoenhancing change appears in the nodule (arrow). D. However, Kupffer-phase imaging shows a defective lesion (arrow) at the corresponding site to the arterial hyperenhancing nodule. US, ultrasonography; $\mathrm{CT}$, computed tomography; MRI, magnetic resonance imaging; LI-RADS (LR), Liver Imaging Reporting and Data System category.

\section{Sonazoid-Enhanced US Features of Hepatic Nodules by CT/ MRI LI-RADS Category}

Sonazoid-enhanced US features of 203 hepatic nodules by CT/MRI LI-RADS category are shown in Table 2. In the AP, most CT/MRI LR-5 (89.6\%) and LR-4 (85.9\%) nodules showed hyperenhancement, while only $57.6 \%$ of LR-3 nodules showed hyperenhancement. The proportion of hypoenhancing nodules in all LR categories was higher in the LP than in the PVP. In the KP, 98.5\% of LR-5 nodules and $92.9 \%$ of LR-4 nodules showed hypoenhancement, while only $75.5 \%$ of LR-3 nodules showed hypoenhancement.

Among the CT/MRI LR-5 nodules, $88.1 \%$ showed in-whole or in-part enhancement. Among CT/MRI LR-5 nodules that showed hyperenhancement or isoenhancement in the AP of CEUS, 59.7\% showed a hypoenhancing change from the PVP, 23.9\% from the LP, and $13.4 \%$ additionally in the KP (Fig. 4). Eight (24.2\%) of the LR-3 nodules, seven $(7.1 \%)$ of the LR-4 nodules, and one (1.5\%) LR-5 


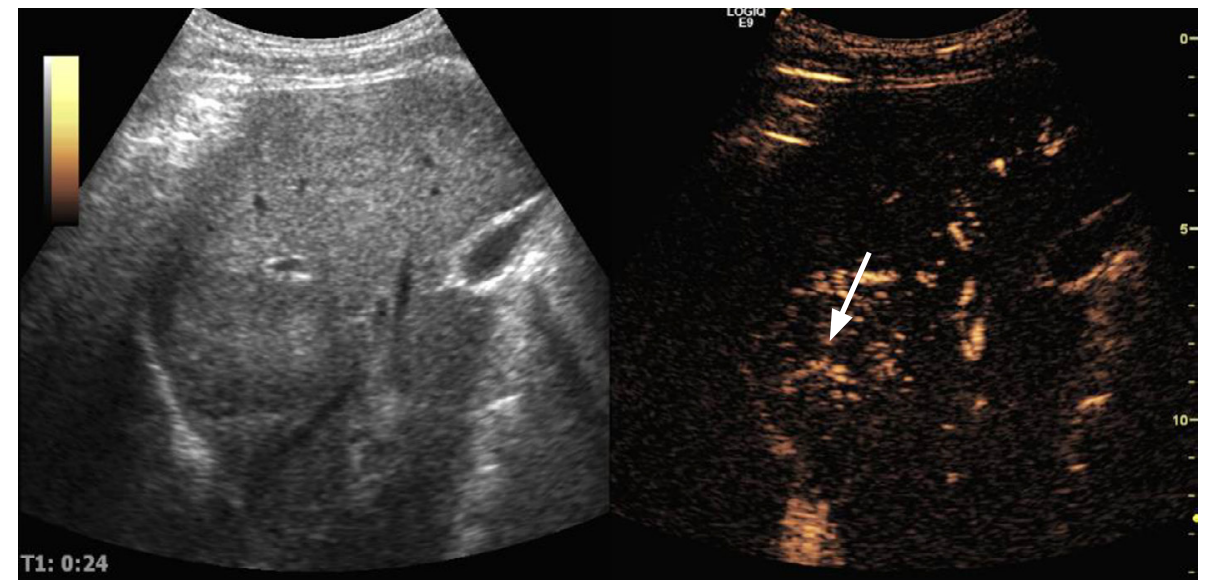

A



C

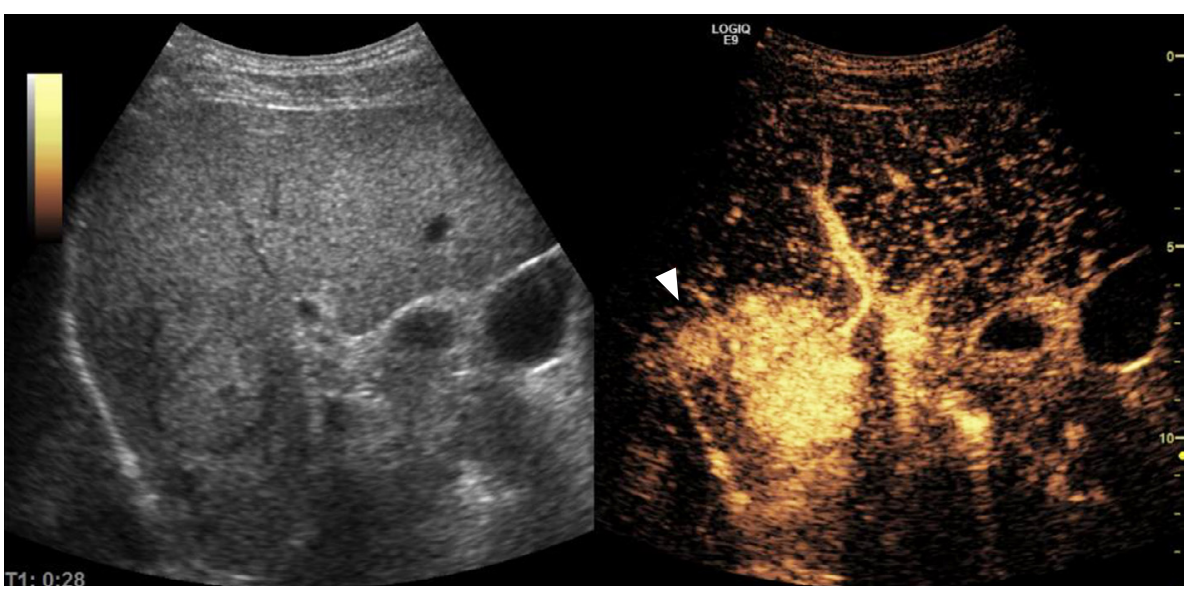

E

nodule did not show washout until the KP.

Two of the four CT/MRI LR-M nodules showed rim APHE. One of the two nodules showed early and marked washout and was considered to be recurrent HCC in a patient who had undergone resection for scirrhous HCC. The other nodule showed late and mild

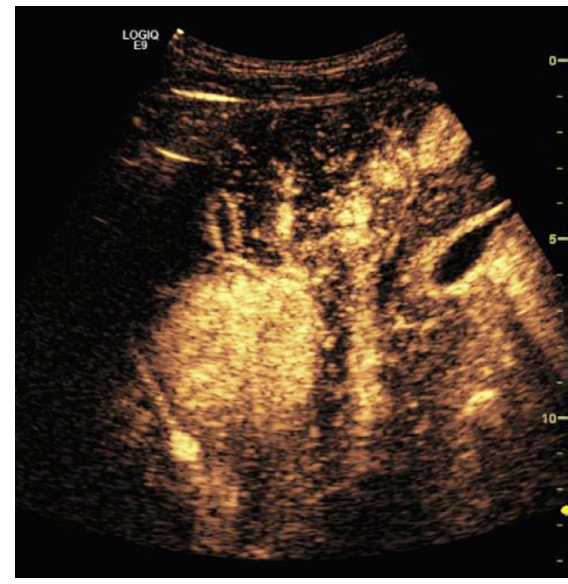

B

Fig. 5. Sonazoid-enhanced US for a CT/ MRI LI-RADS LR-5 nodule with focal nodular hyperplasia confirmed by USguided biopsy in a 68-year-old man with chronic viral hepatitis $B$.

A, B. Arterial phase images show a $4.5-\mathrm{cm}$ hyperenhancing liver nodule with stellate vessels (arrow) in hepatic segment VIII. C, D. The nodule shows subtle hypoenhancement (arrows) from the portal venous phase (C) and lasting until the Kupffer phase (D). E. Nine months later, the nodule has grown to $6.6 \mathrm{~cm}$ and shows extranodular growth (arrowhead) with hyperenhancement in the arterial phase. US, ultrasonography; $\mathrm{CT}$, computed tomography; MRI, magnetic resonance imaging; LI-RADS (LR), Liver Imaging Reporting and Data System category. 


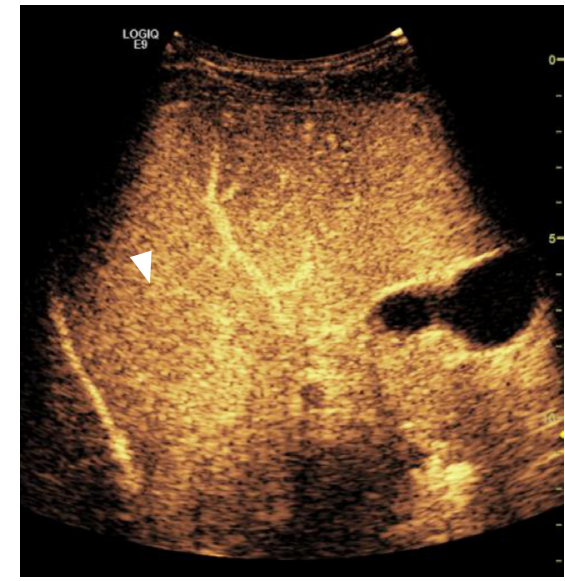

$\mathrm{F}$

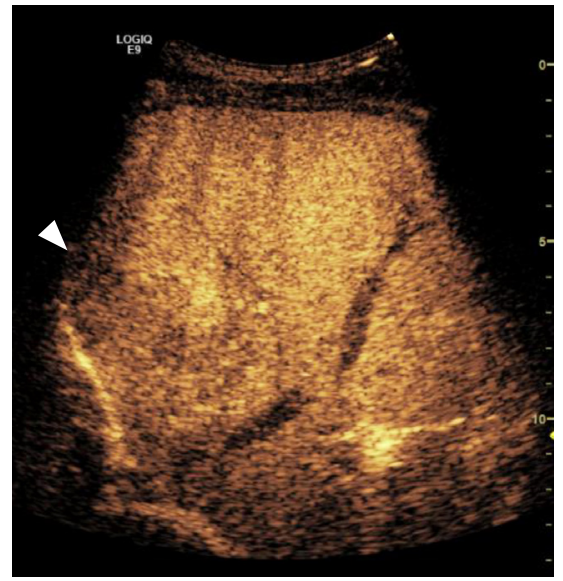

G

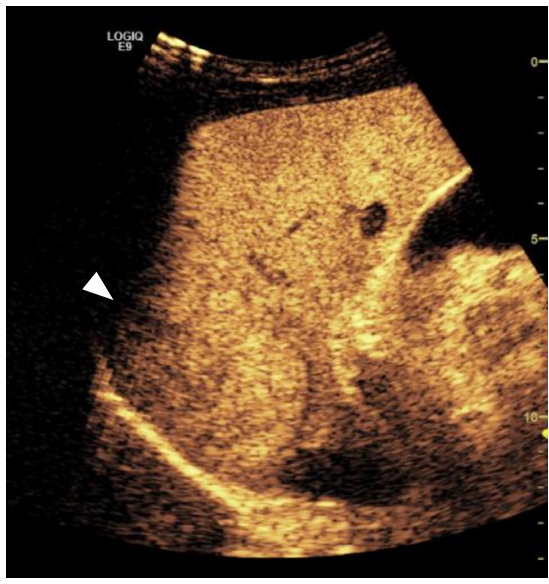

H

Fig. 5. F-H. The nodule shows hypoenhancement (arrowhead) in part from the portal venous phase, lasting until the Kupffer phase. The nodule was finally diagnosed as hepatocellular carcinoma through surgery. US, ultrasonography; CT, computed tomography; MRI, magnetic resonance imaging; LI-RADS (LR), Liver Imaging Reporting and Data System.

\section{Comparison of Sonazoid-Enhanced US Features of CT/MRI} LR-3, LR-4, and LR-5 Nodules

When applying the CEUS LI-RADS major features for 199 hepatic nodules in CT/MRI LR-3, LR-4, and LR-5, APHE and late and mild washout showed significant differences across CT/MRI LI-RADS categories $(\mathrm{P}<0.001)$ (Table 3). APHE and late and mild washout were less frequent in CT/MRI LR-3 nodules than in LR-4 or LR-5 nodules. KP defects also showed significant differences across CT/ MRI LI-RADS categories $(P<0.001)$, and LR-3 nodules had the lowest frequency. KP defects were more frequent than late and mild washout in each CT/MRI LI-RADS category.

\section{Comparison of the Diagnostic Performance of CEUS and Modified CEUS LI-RADS}

For 122 hepatic nodules measuring $1 \mathrm{~cm}$ or more, the diagnostic performance of CEUS and modified CEUS LI-RADS was evaluated (Tables 4, 5). The modified CEUS LI-RADS using KP defects showed higher sensitivity than CEUS LI-RADS ( $83.2 \%$ vs. $74.2 \%, P=0.008$ ). Although the specificity of the modified CEUS LI-RADS was lower than that of CEUS LI-RADS, the difference was not statistically significant (63.6\% vs. $69.7 \%, P=0.500)$. There were no significant differences in accuracy and area under the curve $(P>0.05)$.

\section{Comparison of Nodule Enhancement on MRI HBP and CEUS KP Images}

Table 6 shows a comparison of nodule enhancement on HBP and KP images for CT/MRI LR-3, LR-4, and LR-5 nodules in 193 patients with gadoxetic acid-enhanced MRI. Among a total of 184 hypointense nodules in the HBP, 12 nodules (6.5\%) showed isoenhancement in the KP. In the comparison by each CT/MRI LR category, there was no significant difference in nodule enhancement in the HBP and KP. The proportion of nodules that appeared hypointense in the HBP but without KP defects decreased to $19.2 \%$, $6.3 \%$, and $1.6 \%$ as the CT/MRI LR category increased to LR-3, LR-4, and LR-5, respectively.

\section{Sonazoid-Enhanced US Features of Histologically Diagnosed Hepatic Nodules}

The frequency of HCC among histologically proven hepatic nodules was $42.9 \%$ in $L R-3,87.5 \%$ in LR-4, $85.7 \%$ in LR-5, and $50 \%$ in LR-M of the CT/MRI LR categories (Supplementary Table 2). According to the CEUS/modified CEUS LI-RADS categories, the frequency of HCC was $50 \%$ in LR-3, 66.7\% in LR-4, 85.7\% in LR-5, and none in LR-M - the results of nodule assignments according to CEUS LI-RADS and the modified CEUS LI-RADS were the same.

Except for nodule enhancement on the LP image, there was no significant difference in Sonazoid-enhancement US features between the HCC and non-HCC lesions (Supplementary Table 3). A unique nodule that displayed stellate vessels in the AP and subtle hypoenhancement in the PVP, LP, and KP was diagnosed as a focal nodular hyperplasia-like nodule through US-guided biopsy. However, surgical resection was performed 9 months later due to an increase in size, and the final diagnosis was HCC (Fig. 5).

In the comparison of diagnostic performance for 31 histologically proven lesions, CEUS and the modified CEUS LI-RADS showed higher sensitivity, accuracy, and area under the curve than CT/MRI LI-RADS, but without statistical significance (Supplementary Table 4). Among the eight histologically proven HCCs diagnosed as HCC in CEUS LI-RADS but not in CT/MRI LI-RADS, three had no APHE and the other three had no washout on MRI. The remaining two lesions 
were smaller than $1 \mathrm{~cm}$ on MRI, but measured more than $1 \mathrm{~cm}$ on US.

\section{Discussion}

In the present study, $83.6 \%$ of CT/MRI LI-RADS LR-5 nodules showing hyperenhancement or isoenhancement in the AP showed washout in the PVP or LP on CEUS. An additional $13.4 \%$ of CT/ MRI LR-5 nodules yielded consistent results on CEUS and MRI when the boundary of washout was expanded to the KP. More nodules in the LR-3 category were isoenhancing until the KP than those in the LR-4 or LR-5 of CT/MRI LR categories. In the CT/MRI LR-3, LR-4, and $L R-5$ nodules, KP defects were more frequently seen than late and mild washout. Modified CEUS LI-RADS using KP defects had higher sensitivity for HCC diagnosis than CEUS LI-RADS without significant inferiority in specificity.

Washout might be an important feature used to distinguish malignancy from benignity [26]. A lesion showing sustained enhancement that is equal to or greater than that of the liver in the PVP of CEUS was found to suggest benignity (92\% sensitivity and $93 \%$ specificity) [27]. According to previous studies using KP imaging, however, $10.0 \%-33.3 \%$ of HCCs showed washout only in the KP and not in the late vascular phase $[7,28,29]$. In this study, the modified CEUS LI-RADS using KP defects instead of late and mild washout showed a higher sensitivity for HCC diagnosis than CEUS LI-RADS without significantly compromising the specificity. The application of KP images could be reasonably expected to improve the sensitivity of HCC diagnosis.

In this study, $6.5 \%$ of CT/MRI LR-3, LR-4, and LR-5 nodules showed hypointensity in the HBP but isoenhancement in the KP. Ohama et al. [6] reported that uptake of Sonazoid started to decrease later than that of Gd-EOB-DTPA in stepwise hepatocarcinogenesis, and that hypoenhancement in the KP suggested progressive HCC. In this study, the proportion of nodules that appeared hypointense in the HBP but without a KP defect decreased as the CT/MRI LR category rose. It is difficult to distinguish between DN and early HCC for an isoenhancing nodule in the KP, because Kupffer cells can remain inside both DN and early HCC. Given that all DNs were isoenhancing in the KP compared with $42.1 \%-75.0 \%$ of well-differentiated HCCs $[6,7,10,13,29]$, the possibility of DN can be excluded for hypoenhancing nodules in the KP.

Although this study did not compare a pure blood-pool agent and Sonazoid directly, the CEUS results from the vascular phase using Sonazoid seem to be comparable with those of prior studies using pure blood-pool agents. According to previous reports, washout was observed in $40.0 \%-90.1 \%$ of HCC in CEUS using pure bloodpool agents [30-37], and $75.0 \%-97.2 \%$ of HCC in Sonazoid- enhanced US $[10,28]$. Few studies have investigated whether the hepatic enhancement due to Kupffer cell uptake of microbubbles in the late vascular phase emphasizes washout, and whether there is a difference in washout expression between pure blood-pool agents and Sonazoid. The possibility of pseudo-washout is likely to appear in the KP as well. Therefore, an intra-individual comparison study using these agents would be the logical next step.

This study has several limitations. Firstly, given its retrospective nature, it was subject to selection bias. In many patients, CEUS was performed for pre-treatment assessment before locoregional therapy. The exclusion of strongly hyperechoic nodules in-whole on baseline US could have also caused bias, but it was inevitable considering the issue of pseudoenhancement and false masking of washout. In severe fatty liver, a nodule could also show false washout [19], but there was no such case in this study. A second limitation is that only some lesions had histological diagnoses. Among the histologically proven lesions in this study, HCC was found in $42.9 \%$ of LR-3, $87.5 \%$ of LR- $4,85.7 \%$ of LR-5, and $50 \%$ in LR-M nodules in the CT/ MRI LR categories. To overcome this point, we used the KLCA-NCC 2018 guideline for the imaging diagnosis of HCC as a reference standard to assess the diagnostic performance of CEUS LI-RADS and modified CEUS LI-RADS, considering that the KLCA-NCC 2018 guideline showed higher sensitivity $(79.1 \%$ vs. $68.2 \%, P<0.001)$ without compromising specificity (93.9\% vs. $95.4 \%, P=0.314)$ than CT/MRI LI-RADS v2018 in a recent study [38]. Nevertheless, the specificity of CEUS LI-RADS and the modified CEUS LI-RADS in this study was $69.7 \%$ and $63.6 \%$, respectively, which was disappointing considering that LI-RADS is aimed at high specificity. The characteristics of the KLCA-NCC guideline, which emphasizes specificity rather than sensitivity, and the relatively small size of the 122 lesions (median size, $1.4 \mathrm{~cm}$ ) used to assess diagnostic performance in this study may have affected these results.

In conclusion, the KP best showed hypoenhancing changes in LR-5 lesions and its use is expected to improve the sensitivity for HCC in patients at high risk.

ORCID: Jeong Ah Hwang: https://orcid.org/0000-0002-8012-995X; Woo Kyoung Jeong: https://orcid.org/0000-0002-0676-2116; Ji Hye Min: https://orcid.org/00000002-8496-6771; Yeun-Yoon Kim: https://orcid.org/0000-0003-2018-5332; Nam Hun Heo: https://orcid.org/0000-0001-7278-2214; Hyo Keun Lim: https://orcid.org/00000003-3269-7503

\section{Author Contributions}

Conceptualization: Jeong WK. Data acquisition: Hwang JA, Jeong WK, Lim HK. Data analysis or interpretation: Hwang JA, Jeong WK, Min JH, Kim YY, Heo NH, Lim HK. Drafting of the manuscript: Hwang $J A$, Jeong WK. Critical revision of the manuscript: Jeong WK, Min 
JH, Kim YY, Heo NH, Lim HK. Approval of the final version of the manuscript: all authors.

\section{Conflict of Interest}

Woo Kyoung Jeong serves as Editor for the Ultrasonography, but have no role in the decision to publish this article. All remaining authors have declared no conflicts of interest.

\section{Acknowledgments}

This work was supported by the National Research Foundation of Korea (NRF) grant funded by the Korean government (MSIT) (No. 2019R1G1A1100144).

\section{Supplementary Material}

Supplementary Table 1. Contrast-enhanced ultrasonography performed using Sonazoid protocol (https://doi.org/10.14366/ usg.20120).

Supplementary Table 2. CT/MRI LI-RADS categories for 31 histologically proven lesions (https://doi.org/10.14366/usg.20120).

Supplementary Table 3. Sonazoid-enhanced US features for 31 histologically proven lesions (https://doi.org/10.14366/usg.20120).

Supplementary Table 4. The comparison of diagnostic performance of CT/MRI LI-RADS, CEUS LI-RADS and modified CEUS LI-RADS and for 31 histologically proven lesions (https://doi.org/10.14366/ usg.20120).

\section{References}

1. American College of Radiology. CEUS LI-RADS V2017 [Internet]. Reston, VA: American College of Radiology, 2017 [cited 2020 Aug 5]. Available from: https://www.acr.org/Clinical-Resources/ Reporting-and-Data-Systems/LI-RADS/CEUS-LI-RADS-v2017.

2. Kudo M. Breakthrough imaging in hepatocellular carcinoma. Liver Cancer 2016;5:47-54.

3. Yanagisawa K, Moriyasu F, Miyahara T, Yuki M, lijima H. Phagocytosis of ultrasound contrast agent microbubbles by Kupffer cells. Ultrasound Med Biol 2007;33:318-325.

4. Solbiati L, Tonolini M, Cova L, Goldberg SN. The role of contrastenhanced ultrasound in the detection of focal liver leasions. Eur Radiol 2001;11 Suppl 3:E15-E26.

5. Bartolotta TV, Taibbi A, Midiri M, Lagalla R. Contrast-enhanced ultrasound of hepatocellular carcinoma: where do we stand? Ultrasonography 2019;38:200-214.

6. Ohama H, Imai Y, Nakashima O, Kogita S, Takamura M, Hori M, et al. Images of Sonazoid-enhanced ultrasonography in multistep hepatocarcinogenesis: comparison with Gd-EOB-DTPA-enhanced MRI. J Gastroenterol 2014;49:1081-1093.

7. Inoue T, Kudo M, Maenishi O, Komuta M, Nakashima O, Kojiro M, et al. Value of liver parenchymal phase contrast-enhanced sonography to diagnose premalignant and borderline lesions and overt hepatocellular carcinoma. AJR Am J Roentgenol 2009;192:698705.

8. Inoue T, Hyodo T, Korenaga K, Murakami T, Imai Y, Higaki A, et al. Kupffer phase image of Sonazoid-enhanced US is useful in predicting a hypervascularization of non-hypervascular hypointense hepatic lesions detected on Gd-EOB-DTPA-enhanced MRI: a multicenter retrospective study. J Gastroenterol 2016;51:144-152.

9. Luo W, Numata K, Morimoto M, Kondo M, Takebayashi S, Okada $M$, et al. Focal liver tumors: characterization with 3D perflubutane microbubble contrast agent-enhanced US versus 3D contrastenhanced multidetector CT. Radiology 2009;251:287-295.

10. Hatanaka K, Kudo M, Minami Y, Ueda T, Tatsumi C, Kitai S, et al. Differential diagnosis of hepatic tumors: value of contrast-enhanced harmonic sonography using the newly developed contrast agent, Sonazoid. Intervirology 2008;51 Suppl 1:61-69.

11. Moriyasu F, Itoh K. Efficacy of perflubutane microbubble-enhanced ultrasound in the characterization and detection of focal liver lesions: phase 3 multicenter clinical trial. AJR Am J Roentgenol 2009; 193:86-95.

12. Maruyama H, Takahashi M, Ishibashi H, Okabe S, Yoshikawa M, Yokosuka 0 . Changes in tumor vascularity precede microbubble contrast accumulation deficit in the process of dedifferentiation of hepatocellular carcinoma. Eur J Radiol 2010;75:e102-e106.

13. Korenaga K, Korenaga M, Furukawa M, Yamasaki T, Sakaida I. Usefulness of Sonazoid contrast-enhanced ultrasonography for hepatocellular carcinoma: comparison with pathological diagnosis and superparamagnetic iron oxide magnetic resonance images. J Gastroenterol 2009;44:733-741.

14. Xia Y, Kudo M, Minami Y, Hatanaka K, Ueshima K, Chung H, et al. Response evaluation of transcatheter arterial chemoembolization in hepatocellular carcinomas: the usefulness of sonazoid-enhanced harmonic sonography. Oncology 2008;75 Suppl 1:99-105.

15. Luo W, Numata K, Morimoto M, Oshima T, Ueda M, Okada M, et al. Role of Sonazoid-enhanced three-dimensional ultrasonography in the evaluation of percutaneous radiofrequency ablation of hepatocellular carcinoma. Eur J Radiol 2010;75:91-97.

16. Arita J, Takahashi M, Hata S, Shindoh J, Beck Y, Sugawara Y, et al. Usefulness of contrast-enhanced intraoperative ultrasound using Sonazoid in patients with hepatocellular carcinoma. Ann Surg 2011;254:992-999.

17. Park JH, Park MS, Lee SJ, Jeong WK, Lee JY, Park MJ, et al. Contrastenhanced US with perfluorobutane for hepatocellular carcinoma surveillance: a multicenter diagnostic trial (SCAN). Radiology 
2019;292:638-646.

18. Mandai $M$, Koda M, Matono $T$, Nagahara $T$, Sugihara T, Ueki $M$, et al. Assessment of hepatocellular carcinoma by contrast-enhanced ultrasound with perfluorobutane microbubbles: comparison with dynamic CT. Br J Radiol 2011;84:499-507.

19. Jo PC, Jang HJ, Burns PN, Burak KW, Kim TK, Wilson SR. Integration of contrast-enhanced US into a multimodality approach to imaging of nodules in a cirrhotic liver: how I do it. Radiology 2017;282:317331.

20. Numata K, Luo W, Morimoto M, Kondo M, Kunishi Y, Sasaki T, et al. Contrast enhanced ultrasound of hepatocellular carcinoma. World J Radiol 2010;2:68-82.

21. Lee JY, Minami Y, Choi BI, Lee WJ, Chou YH, Jeong WK, et al. The AFSUMB Consensus statements and recommendations for the clinical practice of contrast-enhanced ultrasound using Sonazoid. Ultrasonography 2020;39:191-220.

22. Kim TK, Noh SY, Wilson SR, Kono Y, Piscaglia F, Jang HJ, et al. Contrast-enhanced ultrasound (CEUS) liver imaging reporting and data system (LI-RADS) 2017: a review of important differences compared to the CT/MRI system. Clin Mol Hepatol 2017;23:280289.

23. Dietrich CF, Dong Y, Kono Y, Caraiani C, Sirlin CB, Cui XW, et al. LIRADS ancillary features on contrast-enhanced ultrasonography. Ultrasonography 2020;39:221-228.

24. American College of Radiology. CT/MRI LI-RADS v2018 [Internet]. Reston, VA: American College of Radiology, 2018 [cited 2020 Aug 5]. Available from: https://www.acr.org/Clinical-Resources/ Reporting-and-Data-Systems/LI-RADS/CT-MRI-LI-RADS-v2018.

25. Korean Liver Cancer Association (KLCA); National Cancer Center (NCC). 2018 Korean Liver Cancer Association-National Cancer Center Korea practice guidelines for the management of hepatocellular carcinoma. Korean J Radiol 2019;20:1042-1113.

26. Yang HK, Burns PN, Jang HJ, Kono Y, Khalili K, Wilson SR, et al. Contrast-enhanced ultrasound approach to the diagnosis of focal liver lesions: the importance of washout. Ultrasonography 2019;38:289-301.

27. Wilson SR, Burns PN. An algorithm for the diagnosis of focal liver masses using microbubble contrast-enhanced pulse-inversion sonography. AJR Am J Roentgenol 2006;186:1401-1412.

28. Takahashi M, Maruyama H, Ishibashi H, Yoshikawa M, Yokosuka O. Contrast-enhanced ultrasound with perflubutane microbubble agent: evaluation of differentiation of hepatocellular carcinoma. AJR Am J Roentgenol 2011;196:W123-W131.

29. Kudo M, Hatanaka K, Inoue T, Maekawa K. Depiction of portal supply in early hepatocellular carcinoma and dysplastic nodule: value of pure arterial ultrasound imaging in hepatocellular carcinoma. Oncology 2010;78 Suppl 1:60-67.

30. Gaiani S, Celli N, Piscaglia F, Cecilioni L, Losinno F, Giangregorio F, et al. Usefulness of contrast-enhanced perfusional sonography in the assessment of hepatocellular carcinoma hypervascular at spiral computed tomography. J Hepatol 2004;41:421-426.

31. Jang HJ, Kim TK, Burns PN, Wilson SR. Enhancement patterns of hepatocellular carcinoma at contrast-enhanced US: comparison with histologic differentiation. Radiology 2007;244:898-906.

32. Jang HJ, Kim TK, Wilson SR. Small nodules $(1-2 \mathrm{~cm})$ in liver cirrhosis: characterization with contrast-enhanced ultrasound. Eur J Radiol 2009;72:418-424.

33. Schellhaas B, Hammon M, Strobel D, Pfeifer L, Kielisch C, Goertz RS, et al. Interobserver and intermodality agreement of standardized algorithms for non-invasive diagnosis of hepatocellular carcinoma in high-risk patients: CEUS-LI-RADS versus MRI-LI-RADS. Eur Radiol 2018;28:4254-4264.

34. Terzi E, lavarone M, Pompili M, Veronese L, Cabibbo G, Fraquelli M, et al. Contrast ultrasound LI-RADS LR-5 identifies hepatocellular carcinoma in cirrhosis in a multicenter restropective study of 1,006 nodules. J Hepatol 2018;68:485-492.

35. Quaia E, D'Onofrio M, Cabassa P, Vecchiato F, Caffarri S, Pittiani $F$, et al. Diagnostic value of hepatocellular nodule vascularity after microbubble injection for characterizing malignancy in patients with cirrhosis. AJR Am J Roentgenol 2007;189:1474-1483.

36. Boozari B, Soudah B, Rifai K, Schneidewind S, Vogel A, Hecker $H$, et al. Grading of hypervascular hepatocellular carcinoma using late phase of contrast enhanced sonography: a prospective study. Dig Liver Dis 2011;43:484-490.

37. Leoni S, Piscaglia F, Granito A, Borghi A, Galassi M, Marinelli S, et al. Characterization of primary and recurrent nodules in liver cirrhosis using contrast-enhanced ultrasound: which vascular criteria should be adopted? Ultraschall Med 2013;34:280-287.

38. Lee S, Kim SS, Chang DR, Kim H, Kim MJ. Comparison of LIRADS 2018 and KLCA-NCC 2018 for noninvasive diagnosis of hepatocellular carcinoma using magnetic resonance imaging. Clin Mol Hepatol 2020;26:340-351. 